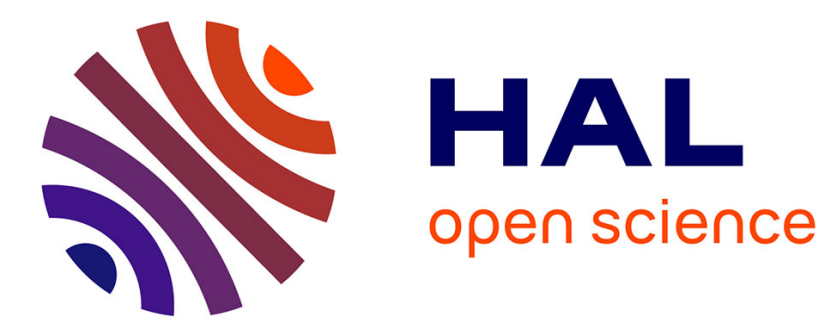

\title{
On a class of integrable systems with a cubic first integral
}

Galliano Valent

\section{To cite this version:}

Galliano Valent. On a class of integrable systems with a cubic first integral. Communications in Mathematical Physics, 2010, 299 (3), pp.631-649. 10.1007/s00220-010-1107-7 . hal-00517631

\section{HAL Id: hal-00517631 \\ https://hal.science/hal-00517631}

Submitted on 15 Sep 2010

HAL is a multi-disciplinary open access archive for the deposit and dissemination of scientific research documents, whether they are published or not. The documents may come from teaching and research institutions in France or abroad, or from public or private research centers.
L'archive ouverte pluridisciplinaire HAL, est destinée au dépôt et à la diffusion de documents scientifiques de niveau recherche, publiés ou non, émanant des établissements d'enseignement et de recherche français ou étrangers, des laboratoires publics ou privés. 
February 2010

\title{
ON A CLASS OF INTEGRABLE SYSTEMS WITH A CUBIC FIRST INTEGRAL
}

\author{
Galliano Valent* \\ * Laboratoire de Physique Théorique et des Hautes Energies \\ Unité associée au CNRS UMR 7589 \\ 2 Place Jussieu, 75251 Paris Cedex 05, France
}

\begin{abstract}
A few years ago Selivanova gave an existence proof for some integrable models, in fact geodesic flows on two dimensional manifolds, with a cubic first integral. However the explicit form of these models hinged on the solution of a nonlinear third order ordinary differential equation which could not be obtained. We show that an appropriate choice of coordinates allows for integration and gives the explicit local form for the full family of integrable systems. The relevant metrics are described by a finite number of parameters and lead to a large class of models mainly on the manifolds $\mathbb{S}^{2}$ and $\mathbb{H}^{2}$. Many of these systems are globally defined and contain as special cases integrable systems due to Goryachev, Chaplygin, Dullin, Matveev and Tsiganov.
\end{abstract}




\section{Introduction}

Let $M$ be a $n$-dimensional smooth manifold with metric $g(X, Y)=g_{i j} X^{i} Y^{j}$ and let $T^{*} M$ be its cotangent bundle with coordinates $(x, P)$, where $P$ is a covector from $T_{x}^{*} M$. Let us recall that $T^{*} M$ is a smooth symplectic $2 n$-manifold with respect to the standard 2 -form $\omega=d P_{i} \wedge d x^{i}$ which induces the Poisson bracket

$$
\{f, g\}=\sum_{i=1}^{n}\left(\frac{\partial f}{\partial x^{i}} \frac{\partial g}{\partial P_{i}}-\frac{\partial f}{\partial P_{i}} \frac{\partial g}{\partial x^{i}}\right) .
$$

In $T^{*} M$ the geodesic flow is defined by the Hamiltonian

$$
H=K+V, \quad K=\frac{1}{2} \sum_{i, j=1}^{n} g^{i j}(x) P_{i} P_{j}, \quad V=V(x),
$$

where $g^{i j}$ is the inverse metric of $g_{i j}$.

An "observable" $f: T^{*} M \rightarrow \mathbb{R}$, which can be written locally

$$
f=\sum_{i_{1}+\cdots+i_{n} \leq m} f^{i_{1}, \cdots, i_{n}}(x) P_{i_{1}} \cdots P_{i_{n}}, \quad \#(f)=m,
$$

is a constant of motion iff $\{H, f\}=0$. A hamiltonian system is said to be integrable in Liouville sense if there exist $n$ constants of motion (including $H$ ) generically independent and in pairwise involution with respect to the Poisson bracket.

In what follows we will deal exclusively with integrable systems defined on two dimensional manifolds: in this case an integrable system is just made out of two independent observables $H$ and $Q$ with $\{H, Q\}=0$.

The general line of attack of this problem is based on the integer $m=\#(Q)$. For $m=1 M$ is a surface of revolution and for $m=2 M$ is a Liouville surface [3].

For higher values of $m$ only particular examples have been obtained, some of which in explicit form. For $M=\mathbb{S}^{2}$ and $m=3$ the oldest explicit examples (early twentieth century) were due to Goryachev and Chaplygin on the one hand and to Chaplygin on the other hand (see [2][p. 483] and [10] for the detailed references). On the same manifold with $m=4$ there is the famous Kovalevskaya system [7] and some extension due to Goryachev (see [9] for the reference).

More recently there was a revival of this subject due to several existence theorems due to Selivanova and Kiyohara. Selivanova studied integrable systems both for $m=3$ in [8] and for $m=4$ in [9] and Kiyohara for any $m \geq 3$ in [6]. As observed by Kiyohara, for $m=3$ the two classes of models are markedly different. In the last years several new explicit examples for $m=3$ were given by Dullin and Matveev [4] and Tsiganov [10].

In this work we will focus on Selivanova's integrable systems with a cubic first integral discussed in [8]. A natural question raised by her existence theorems is the possibility of a constructive approach. According to the coordinates chosen one has to solve either a third order nonlinear ODE, as in [8], or a fourth order one as in Tsiganov's work [10].

We will show that one can avoid to solve these ODE: an appropriate coordinates choice allows to get locally the explicit form of the full family of integrable systems, described by finitely many parameters. The final step is then, according to the values taken by the parameters, to determine the manifold $M$ (mainly $\mathbb{S}^{2}$ and $\mathbb{H}^{2}$ ) on which the systems are defined and whether the observables $H$ and $Q$ are globally defined on $M$. 
The plan of the article is the following: in Section 2 we consider the class of models analyzed by Selivanova with the following leading terms for the cubic observable:

$$
Q=p P_{\phi}^{3}+2 q K P_{\phi}+\cdots, \quad p \in \mathbb{R}, \quad q \geq 0,
$$

and the general differential system resulting of $\{H, Q\}=0$ is given.

In Section 3 we first integrate the special case where $q=0$ : the differential system is reduced to a second order non-linear ODE. The global issues are then discussed.

In Section 4 we consider the general case $q>0$. Here we have linearized, by an appropriate choice of the coordinates, the possibly non-linear ODE encountered in other approaches.

In Section 5, with the explicit local form of the metric, it is then possible (but lengthy because an enumeration of cases is required) to determine on which manifolds the metric is defined. We have checked that all the explicit integrable models given in the literature are indeed recovered as special cases.

\section{Cubic first integral}

The general structure of the integrable system, laid down by Selivanova [8], is the following: one starts from the hamiltonian (1) with

$$
K=\frac{1}{2}\left(P_{\theta}^{2}+a(\theta) P_{\phi}^{2}\right), \quad V=f(\theta) \cos \phi+g(\theta), \quad f(\theta) \not \equiv 0,
$$

and the cubic observable

$$
Q=Q_{3}+Q_{1}
$$

with

$$
\left\{\begin{array}{l}
Q_{3}=p P_{\phi}^{3}+2 q K P_{\phi}, \quad p \in \mathbb{R}, q \geq 0 \\
Q_{1}=\chi(\theta) \sin \phi P_{\theta}+(\beta(\theta)+\gamma(\theta) \cos \phi) P_{\phi}
\end{array}\right.
$$

Lemma 1 The constraint $\{H, Q\}=0$ is equivalent to the following differential system:

$$
\begin{gathered}
\chi \dot{f}=\gamma f, \quad \chi \dot{g}=\beta f, \quad\left(\cdot=D_{\theta}\right), \\
\text { (b) } \quad \dot{\chi}=-q f, \quad \dot{\beta}=2 q \dot{g}, \quad \dot{\gamma}+\chi a=2 q \dot{f}, \quad a \gamma+\chi \frac{\dot{a}}{2}=3(p+q a) f .
\end{gathered}
$$

Proof: The relation $\{H, Q\}=0$ splits into three constraints

$$
\left\{K, Q_{3}\right\}=0, \quad\left\{K, Q_{1}\right\}+\left\{V, Q_{3}\right\}=0, \quad\left\{V, Q_{1}\right\}=0 .
$$

The first is identically true, the second one is equivalent to the relations (5b) while the last one is equivalent to $(5 \mathrm{a})$.

The special case $q=0$ is rather difficult to obtain as the limit of the general case $q \neq 0$, so we will first work it out completely. 


\section{The integrable system for $q=0$}

\subsection{Local analysis}

We can take $p=1$ and obvious integrations give

$$
\chi=\chi_{0}>0, \quad \beta=\beta_{0} \in \mathbb{R}, \quad \gamma=\chi_{0} \frac{\dot{f}}{f}, \quad \dot{g}=\frac{\beta_{0}}{\chi_{0}} f, \quad a=-\frac{\dot{\gamma}}{\chi_{0}},
$$

and the last equation

$$
\ddot{\gamma}+2 \frac{\dot{f}}{f} \dot{\gamma}+6 f=0
$$

An appropriate choice of coordinates does simplify matters:

Lemma 2 The differential equation for $u=\dot{f}$ as a function of the variable $x=f$ is given by

$$
u\left(\frac{u u^{\prime}}{x}\right)^{\prime}+c x=0, \quad c=\frac{6}{\chi_{0}}>0 . \quad\left({ }^{\prime}=D_{x}\right) .
$$

Proof: The relations in (7) become

$$
g^{\prime}=\frac{\beta_{0}}{\chi_{0}} \frac{x}{u}, \quad \gamma=\chi_{0} \frac{u}{x}, \quad a=-u\left(\frac{u}{x}\right)^{\prime},
$$

and (8) gives (9).

The solution of this ODE follows from

Lemma 3 The general solution of (9) is given by

$$
u=-\frac{\zeta^{2}+c_{0}}{2 c}
$$

with

$$
\zeta^{3}+3 c_{0} \zeta-2 \rho=0, \quad 2\left(\rho-\rho_{0}\right)=3 c^{2} x^{2},
$$

and integration constants $\left(\rho_{0}, c_{0}\right)$.

Proof: Let us define $\zeta^{\prime}=-c x / u$. This allows a first integration of (9), giving $\frac{u u^{\prime}}{x}=\zeta$. From this we deduce

$$
c u^{\prime}=-\zeta \zeta^{\prime} \quad \Longrightarrow \quad 2 c u=-\zeta^{2}-c_{0},
$$

which in turn implies

$$
\left[\zeta^{2}+c_{0}\right] \zeta^{\prime}=2 c^{2} x \quad \Longrightarrow \quad \zeta^{3}+3 c_{0} \zeta-2 \rho=0
$$

which concludes the proof.

It is now clear that the initial coordinates $(\theta, \phi)$ chosen on $S^{2}$ will not lead, at least generically, to a simple form of the hamiltonian! To achieve a real simplification for the observables the symplectic coordinates change $\left(\theta, \phi, P_{\theta}, P_{\phi}\right) \rightarrow\left(\zeta, \phi, P_{\zeta}, P_{\phi}\right)$ gives: 
Theorem 1 Locally, the integrable system has for explicit form

$$
\left\{\begin{array}{l}
H=\frac{1}{2}\left(F P_{\zeta}^{2}+\frac{G}{4 F} P_{\phi}^{2}\right)+\lambda \sqrt{F} \cos \phi+\mu \zeta, \\
Q=P_{\phi}^{3}-2 \lambda\left(\sqrt{F} \sin \phi P_{\zeta}+(\sqrt{F})^{\prime} \cos \phi P_{\phi}\right)-2 \mu P_{\phi},
\end{array} \quad\left({ }^{\prime}=D_{\zeta}\right)\right.
$$

with

$$
F=-2 \rho_{0}+3 c_{0} \zeta+\zeta^{3}, \quad G=9 c_{0}^{2}+24 \rho_{0} \zeta-18 c_{0} \zeta^{2}-3 \zeta^{4}
$$

and real parameters $(\lambda, \mu)$.

Proofs: One may obtain these formulas by elementary computations, some scalings of the parameters and $\left(\chi_{0} \rightarrow \lambda, \beta_{0} \rightarrow-\mu\right)$.

Alternatively, one can check that (15) implies the relations

$$
G^{\prime}=-12 F, \quad G=F^{2}-2 F F^{\prime \prime},
$$

which allows for a direct check of $\{H, Q\}=0$. As proved in [8] this system does not exhibit any linear or quadratic constant of motion and $(H, Q)$ are algebraically independent.

\subsection{The manifolds}

We are now in position to analyze the global geometric aspects related to the metric ${ }^{1}$

$$
g=\frac{d \zeta^{2}}{F}+\frac{4 F}{G} d \phi^{2}, \quad \phi \in[0,2 \pi) .
$$

One has first to impose the positivity of both $F$ and $G$ for this metric to be riemannian. This gives for $\zeta$ some interval $I$ whose end-points are possible singularities of the metric. To ascertain that the metric is defined on some manifold one has to ensure that these singularities are apparent ones and not true curvature singularities by techniques developed in [5].

Let us define, for the cubic $F$, its discriminant $\Delta=c_{0}^{3}+\rho_{0}^{2}$.

Theorem 2 The metric (17):

(i) Is defined, for $\Delta<0$, on $\mathbb{S}^{2}$ iff

$$
F=\left(\zeta-\zeta_{0}\right)\left(\zeta-\zeta_{1}\right)\left(\zeta-\zeta_{2}\right), \quad \zeta_{0}<\zeta<\zeta_{1}<\zeta_{2}
$$

The change of coordinates

$$
\operatorname{sn}\left(u, k^{2}\right)=\sqrt{\frac{\zeta-\zeta_{0}}{\zeta_{1}-\zeta_{0}}}, \quad u \in(0, K), \quad k^{2}=\frac{\zeta_{1}-\zeta_{0}}{\zeta_{2}-\zeta_{0}} \in(0,1),
$$

gives for integrable system ${ }^{2}$

$$
\left\{\begin{array}{l}
H=\frac{1}{2}\left(P_{u}^{2}+\frac{D(u)}{s^{2} c^{2} d^{2}} P_{\phi}^{2}\right)+\lambda k^{2} s c d \cos \phi+\mu k^{2} s^{2} \\
Q=4 P_{\phi}^{3}-\lambda\left(\sin \phi P_{u}+\frac{(s c d)^{\prime}}{s c d} \cos \phi P_{\phi}\right)-2 \mu P_{\phi} \\
D(u)=\left(1-k^{2} s^{4}\right)^{2}-4 k^{2} s^{4} c^{2} d^{2}>0
\end{array}\right.
$$

\footnotetext{
${ }^{1}$ As usual we identify the points with $\phi=0$ and with $\phi=2 \pi$.

${ }^{2}$ We use the shorthand notation: $s, c, d$ respectively for $\operatorname{sn}\left(u, k^{2}\right), \operatorname{cn}\left(u, k^{2}\right), \operatorname{dn}\left(u, k^{2}\right)$.
} 
(ii) Is defined, for $\Delta=0$, on $\mathbb{H}^{2}$ iff

$$
F=\left(\zeta-\zeta_{1}\right)^{2}\left(\zeta+2 \zeta_{1}\right), \quad-2 \zeta_{1}<\zeta<\zeta_{1}, \quad\left(\zeta_{1}>0\right) .
$$

The change of coordinates

$$
\zeta=\zeta_{1}\left(-2+3 \tanh ^{2} u\right), \quad u \in(0,+\infty),
$$

gives for integrable system ${ }^{3}$

$$
\left\{\begin{array}{l}
H=\frac{1}{2}\left(P_{u}^{2}+\frac{\left(1+3 T^{2}\right)}{S^{2}} P_{\phi}^{2}\right)+\lambda T\left(1-T^{2}\right) \cos \phi+\mu T^{2} \\
Q=4 P_{\phi}^{3}-\lambda\left(\sin \phi P_{u}+\frac{1-3 T^{2}}{T} \cos \phi P_{\phi}\right)-2 \mu P_{\phi}
\end{array}\right.
$$

(iii) Is not defined on any manifold for $\Delta>0$.

Proof of (i): If $\Delta<0$ the cubic $F$ has three simple real roots $\zeta_{0}<\zeta_{1}<\zeta_{2}$. If we take $\zeta \in\left(\zeta_{2},+\infty\right)$ then $F$ is positive. The relation $G^{\prime}=-12 F$ shows that in this interval $G$ is decreasing from $G\left(\zeta_{2}\right)=F^{\prime 2}\left(\zeta_{2}\right)>0$ to $-\infty$ and will vanish for some $\widehat{\zeta}>\zeta_{2}$. Hence to ensure positivity for $F$ and $G$ we must restrict $\zeta$ to the interval $\left(\zeta_{2}, \widehat{\zeta}\right)$. Since at $\zeta=\widehat{\zeta}$ the function $F$ does not vanish while $G$ does, this point is a curvature singularity and the metric cannot be defined on a manifold.

The positivity of $F$ is also ensured if we take $\zeta \in\left(\zeta_{0}, \zeta_{1}\right)$. In this interval $G$ decreases monotonously from $G\left(\zeta_{0}\right)$ to $G\left(\zeta_{1}\right)=F^{2}\left(\zeta_{1}\right)>0$. Let us analyze the singularities at the end points. For $\zeta$ close to $\zeta_{0}$ we have for approximate metric

$$
g \approx \frac{4}{F^{\prime}\left(\zeta_{0}\right)}\left[\frac{d \zeta^{2}}{4\left(\zeta-\zeta_{0}\right)}+\frac{F^{2}\left(\zeta_{0}\right)}{G\left(\zeta_{0}\right)}\left(\zeta-\zeta_{0}\right) d \phi^{2}\right]
$$

The relation (16) gives $G\left(\zeta_{0}\right)=F^{2}\left(\zeta_{0}\right)$, so the change of variable $\rho=\sqrt{\zeta-\zeta_{0}}$ allows to write

$$
g \approx \frac{4}{F^{\prime}\left(\zeta_{0}\right)}\left(d \rho^{2}+\rho^{2} d \phi^{2}\right)
$$

which shows that $\rho=0$ is an apparent singularity, called a "nut" in [5]. It is a shortcoming of the polar coordinates used and is removed by switching back to cartesian coordinates. So the point $\zeta=\zeta_{0}$ can be added to the manifold and similarly we can add $\zeta=\zeta_{1}$. Geometrically the points $\zeta_{0}$ and $\zeta_{1}$ are the poles of the manifold which is therefore $\mathbb{S}^{2}$. Each "nut" contributes by 1 to the Euler characteristic [5] giving $\chi=2$ as it should. Then, using the change of variable (18), it is a routine exercise in elliptic functions theory to operate the symplectic coordinates change $\left(\zeta, \phi, P_{\zeta}, P_{\phi}\right) \rightarrow\left(u, \phi, P_{u}, P_{\phi}\right)$ which, after several scalings of the observables and of their parameters, gives (19). The strict positivity of $D$ for $u \in[0, K]$ follows from the strict positivity of $G$ for $\zeta \in\left[\zeta_{0}, \zeta_{1}\right]$.

Let us notice that one can also, by direct computation, check that $\{H, Q\}=0$ from the formulas given in (19).

\footnotetext{
${ }^{3}$ We use the shorthand notation $S, C, T$ respectively for $\sinh u, \cosh u, \tanh u$.
} 
Proof of (ii): In this case we have

$$
F=\left(\zeta+2 \zeta_{1}\right)\left(\zeta-\zeta_{1}\right)^{2}, \quad G=-3\left(\zeta+3 \zeta_{1}\right)\left(\zeta-\zeta_{1}\right)^{3}, \quad \zeta_{1}=-\rho_{0}^{1 / 3} .
$$

For $\zeta_{1}<0$ the positivity of $F$ implies $\zeta \in\left(2\left|\zeta_{1}\right|,+\infty\right)$ and $G$ decreases and vanishes for $\widehat{\zeta}=3\left|\zeta_{1}\right|$ leading to a curvature singularity. The case $\zeta_{1}=0$ is also excluded since then $G \leq 0$ and the remaining case is $\zeta_{1}>0$. The positivity of $F$ and $G$ requires $\zeta \in\left(-2 \zeta_{1}, \zeta_{1}\right)$. The singularity structure is most conveniently discussed thanks to the coordinates change (20) which brings the metric to the form

$$
g=\frac{4}{3 \zeta_{1}}\left\{d u^{2}+\frac{\sinh ^{2} u}{1+3 \tanh ^{2} u} d \phi^{2}\right\}, \quad u \in(0,+\infty),
$$

from which we conclude that the manifold is $\mathbb{H}^{2}$. Then starting from (14), the symplectic change of coordinates $\left(\zeta, \phi, P_{\zeta}, P_{\phi}\right) \rightarrow\left(u, \phi, P_{u}, P_{\phi}\right)$, and some scalings, gives (21).

Proof of (iii): For $\Delta>0$ the cubic $F$ has a single real zero $\zeta_{0}$. The positivity of $F$ requires that $\zeta \in\left(\zeta_{0},+\infty\right)$. Since $G^{\prime}=-12 F$ the function $G$ decreases from $G\left(\zeta_{0}\right)$ to $-\infty$. Since $G\left(\zeta_{0}\right)>0$ there exists $\widehat{\zeta}>\zeta_{0}$ for which $G(\widehat{\zeta})=0$. So positivity restricts $\zeta \in\left(\zeta_{0}, \widehat{\zeta}\right)$ and $\widehat{\zeta}$ is a curvature singularity showing that the metric cannot be defined on a manifold.

\section{Remarks:}

1. The integrable system (21) corresponds to the limit of (19) when $\zeta_{2} \rightarrow \zeta_{1}$ or $k^{2} \rightarrow 1$. Then the elliptic functions degenerate into hyperbolic functions. Let us emphasis that in this limit the observables behave smoothly while the manifold changes drastically .

2. In [8] Selivanova proved an existence theorem for an integrable system on $S^{2}$ with a cubic observable (case (i) of Theorem 1.1). Her observables are

$$
\begin{aligned}
& H=\frac{\psi^{\prime 2}(y)}{2}\left(P_{y}^{2}+P_{\phi}^{2}\right)+\frac{\psi^{\prime 2}(y)}{2}\left(\psi(y)-\psi^{\prime \prime}(y)\right) \cos \phi, \quad \quad\left({ }^{\prime}=D_{y}\right), \\
& Q=P_{\phi}^{3}-\frac{3}{2} \psi^{\prime}(y) \sin \phi P_{y}+\frac{3}{2} \psi(y) \cos \phi P_{\phi},
\end{aligned}
$$

where $\psi(y)$ is a solution of the ODE

$$
\psi^{\prime} \psi^{\prime \prime \prime}=\psi \psi^{\prime \prime}-2 \psi^{\prime \prime 2}+\psi^{\prime 2}+\psi^{2}, \quad \psi(0)=0, \psi^{\prime}(0)=1, \psi^{\prime \prime}(0)=\tau .
$$

Comparing (25) and (14) for $\beta=0$ makes it obvious that we are dealing with the same integrable system, up to a local diffeomorphism, which is

$$
\psi(y)=-\frac{\left(\zeta^{2}+c_{0}\right)}{2 \sqrt{F}}, \quad \frac{\sqrt{G}}{F} d \zeta= \pm \sqrt{3} d y
$$

We have checked that the ODE (26) is a consequence of the relations (27) and (16). We see clearly that Selivanova's choice of the coordinate $y$ led to a complicated ODE, very difficult to solve; in fact one should rather find coordinates such that the ODE becomes tractable.

Now that we have obtained two integrable systems: the first on $\mathbb{S}^{2}$ and the second one on $\mathbb{H}^{2}$, let us examine the global properties of the observables. 


\subsection{Global structure}

We will give some details in this section, that will be useful to shorten similar proofs needed in the next sections.

Theorem 3 The integrable system given by (19) is globally defined on $M=\mathbb{S}^{2}$.

Proof: Its potential is

$$
V=\lambda k^{2} s c d \cos \phi+\mu k^{2} s^{2} .
$$

The variable $u \in(0, K)$ and $\{u=0, u=K\}$ are the poles of the sphere. The potential is continuously differentiable for all $u \in[0, K]$. For the observable $Q$ there are apparent singularities at the poles since we have

$$
\frac{(s c d)^{\prime}}{s c d} \sim \frac{1}{u} \quad u \rightarrow 0, \quad \frac{(s c d)^{\prime}}{s c d} \sim \frac{1}{(K-u)} \quad u \rightarrow K .
$$

In fact we need to express $Q$ in terms of globally defined quantities, namely the isometries generators in $T^{*} M$.

The sphere $x_{1}^{2}+x_{2}^{2}+x_{3}^{2}=1$ in the charts

$$
x_{1}=\sin \theta \cos \phi \quad x_{2}=\sin \theta \sin \phi \quad x_{3}= \pm \cos \theta, \quad \theta \in(0, \pi) \quad \phi \in[0,2 \pi),
$$

has for canonical metric

$$
g_{0}\left(\mathbb{S}^{2}, \operatorname{can}\right)=d x_{1}^{2}+d x_{2}^{2}+d x_{3}^{2}=d \theta^{2}+\sin ^{2} \theta d \phi^{2},
$$

to be compared to the metric in (19):

$$
g=d u^{2}+\frac{s^{2} c^{2} d^{2}}{D} d \phi^{2} .
$$

As is well known these two metrics must be conformally related. Indeed defining the correspondence $\theta \rightarrow u$ by

$$
\frac{\theta^{\prime}}{\sin \theta}=\frac{\sqrt{D}}{s c d}, \quad \theta \in(0, \pi) \quad \rightarrow \quad u \in(0, K)
$$

which integrates up to

$$
\tan \frac{\theta}{2}=\frac{s}{c} H(u) \quad H(u)=\exp \left(-\int_{u}^{K} \frac{\sqrt{D}-d^{2}}{s c d} d \tau\right)
$$

we get the relation

$$
g=\Omega g_{0}\left(\mathbb{S}^{2}, \mathrm{can}\right), \quad \Omega=\frac{1}{\theta^{\prime 2}}=\frac{d^{2}\left(c^{2}+s^{2} H^{2}\right)^{2}}{4 H^{2} D} .
$$

The functions $H$ and $\Omega$ are $C^{\infty}$ for $u \in[0, K]$ and strictly positive, showing again that the manifold is indeed $\mathbb{S}^{2}$.

Let us define the $s o(3)$ generators in $T^{*} \mathbb{S}^{2}$ :

$$
L_{1}=\frac{\sin \phi}{\theta^{\prime}} P_{u}+\frac{\cos \phi}{\tan \theta} P_{\phi}, \quad L_{2}=-\frac{\cos \phi}{\theta^{\prime}} P_{u}+\frac{\sin \phi}{\tan \theta} P_{\phi}, \quad L_{3}=P_{\phi} .
$$


The integrable system becomes

$$
\left\{\begin{array}{l}
H=\frac{\theta^{\prime 2}}{2}\left(L_{1}^{2}+L_{2}^{2}+L_{3}^{2}\right)+\lambda k^{2} s c d \cos \phi+\mu k^{2} s^{2} \\
Q=4 L_{3}^{3}-\lambda\left(\theta^{\prime} L_{1}+\rho \cos \phi L_{3}\right)-2 \mu L_{3}, \quad \rho=\frac{(s c d)^{\prime}-\cos \theta \sqrt{D}}{s c d} .
\end{array}\right.
$$

From the previous discussion $\theta^{\prime}$ is $C^{\infty}$ on $[0, K]$. The function $\rho$ is $C^{\infty}$ on $(0, K)$ and the series expansions close to the poles

$$
\rho(u)=2\left(H(0)^{2}-1-k^{2}\right) u+O\left(u^{3}\right) \quad \rho(u)=-2 k^{2}(K-u)+O\left((K-u)^{3}\right)
$$

show that it remains also $C^{\infty}$ through them. Hence we conclude that this integrable system is globally defined on $\mathbb{S}^{2}$.

\section{Remark:}

It is important to observe that the Hamiltonian vector field

$$
v_{H}=\frac{\partial H}{\partial p^{i}} \frac{\partial}{\partial x^{i}}-\frac{\partial H}{\partial x^{i}} \frac{\partial}{\partial p_{i}}
$$

which has for explicit expression

$$
\begin{aligned}
v_{H}= & -\theta^{\prime}\left[\theta^{\prime \prime}\left(P_{\theta}^{2}+\frac{P_{\phi}^{2}}{\sin ^{2} \theta}\right)-\frac{\cos \theta \theta^{\prime 2} P_{\phi}^{2}}{\sin ^{3} \theta}\right] \frac{\partial}{\partial P_{u}}-\frac{\partial V}{\partial u} \frac{\partial}{\partial P_{u}}-\frac{\partial V}{\partial \phi} \frac{\partial}{\partial P_{\phi}} \\
& +\theta^{\prime 2}\left(P_{u} \frac{\partial}{\partial u}+\frac{P_{\phi}}{\sin ^{2} \theta} \frac{\partial}{\partial \phi}\right)
\end{aligned}
$$

must be defined everywhere, to avoid a singular Hamiltonian flow. This requires not only the continuity of $\theta^{\prime}$ and $V$ but also their differentiabilty. This observation will have important consequences for some models discussed in section 5 .

Let us now prove

Theorem 4 The integrable system (21) is globally defined on $M=\mathbb{H}^{2}$.

Proof: Its potential is

$$
V=\lambda T\left(1-T^{2}\right) \cos \phi+\mu T^{2}, \quad T=\tanh u, \quad u \in[0,+\infty),
$$

and is differentiable for all values of $u$ and $\phi$, particularly at the "nut" $u=0$.

The hyperbolic plane

$$
\mathbb{H}^{2}: \quad\left\{x_{1}^{2}+x_{2}^{2}-x_{3}^{2}=-1, \quad\left(x_{1}, x_{2}\right) \in \mathbb{R}^{2} \quad x_{3} \geq 1\right\},
$$

in the chart

$$
x_{1}=\sinh \chi \cos \phi \quad x_{2}=\sinh \chi \sin \phi \quad x_{3}=\cosh \chi, \quad \chi \in[0,+\infty), \quad \phi \in[0,2 \pi)
$$

has for canonical metric

$$
g_{0}\left(\mathbb{H}^{2}, \text { can }\right)=d x_{1}^{2}+d x_{2}^{2}-d x_{3}^{2}=d \chi^{2}+\sinh ^{2} \chi d \phi^{2} .
$$


The metric of $(21)$ is

$$
g=d u^{2}+\frac{\sinh ^{2} u}{1+3 \tanh ^{2} u} d \phi^{2},
$$

which must be conformal to $g_{0}$. Defining the correspondence $\chi \rightarrow u$ by

$$
\frac{\chi^{\prime}}{\sinh \chi}=\frac{\sqrt{1+4 \sinh ^{2} u}}{\sinh u \cosh u}, \quad \chi \in[0,+\infty) \quad \rightarrow \quad u \in[0,+\infty)
$$

which integrates up to

$$
\tanh (\chi / 2)=\tanh u H(u) \quad H(u)=\exp \left(-\int_{u}^{+\infty} \frac{\left(\sqrt{1+4 \sinh ^{2} \tau}-1\right)}{\sinh \tau \cosh \tau} d \tau\right)
$$

we get

$$
g=\Omega g_{0}\left(\mathbb{H}^{2}, \text { can }\right) \quad \Omega=\frac{\cosh ^{2} u\left(1-\tanh ^{2} u H^{2}(u)\right)^{2}}{4\left(1+3 \tanh ^{2} u\right) H^{2}(u)} .
$$

The function $H$, which can be expressed in terms of elementary functions, is $C^{\infty}$ for $u \geq 0$, ensuring that the conformal factor $\Omega(u)$ is also $C^{\infty}$. Its behaviour at infinity is

$$
H(u)=1-e^{-u}+O\left(e^{-2 u}\right) \quad \Longrightarrow \quad \Omega(u)=\frac{1}{16}\left(1+O\left(e^{-u}\right)\right)
$$

hence $\Omega$ never vanishes. This gives another proof that the metric (33) does live on $\mathbb{H}^{2}$.

Following the same line as previously, let us define the generators of the so(2,1) Lie algebra in $T^{*} \mathbb{H}^{2}$

$$
M_{1}=\sin \phi P_{u}+\frac{\cos \phi}{\tanh u} P_{\phi}, \quad M_{2}=\cos \phi P_{u}-\frac{\sin \phi}{\tanh u} P_{\phi}, \quad M_{3}=P_{\phi} .
$$

The observables (21) become

$$
\left\{\begin{array}{l}
H=M_{1}^{2}+M_{2}^{2}-\left(1-\frac{3}{C^{2}}\right) M_{3}^{2}+\lambda T\left(1-T^{2}\right) \cos \phi+\mu T^{2}, \\
Q=4 M_{3}^{3}-\lambda\left(M_{1}-3 T \cos \phi M_{3}\right)-2 \mu M_{3},
\end{array}\right.
$$

showing that this sytem is globally defined on $\mathbb{H}^{2}$.

\section{The integrable system for $q>0$}

As already observed, if one insists in working with the variable $\theta$, the differential system (5) can be reduced either to a third order [8] or to a fourth order [10] non-linear ODE. The key to a full integration of this system is again an appropriate choice of coordinates on the manifold.

Theorem 5 Locally, the integrable system $(H, Q)$ has for explicit form

$$
\left\{\begin{array}{l}
H=\frac{1}{2 \zeta}\left(F P_{\zeta}^{2}+\frac{G}{4 F} P_{\phi}^{2}\right)+\frac{\sqrt{F}}{2 q \zeta} \cos \phi+\frac{\beta_{0}}{2 q \zeta}, \\
Q=p P_{\phi}^{3}+2 q H P_{\phi}-\sqrt{F} \sin \phi P_{\zeta}-(\sqrt{F})^{\prime} \cos \phi P_{\phi},
\end{array} \quad\left({ }^{\prime}=D_{\zeta}\right),\right.
$$


with the polynomials

$$
F=c_{0}+c_{1} \zeta+c_{2} \zeta^{2}+\frac{p}{q} \zeta^{3}, \quad G=F^{2}-2 F F^{\prime \prime}
$$

Proofs: Starting from (5) the functions $\beta$ and $g$ are easily determined to be

$$
\beta=\frac{\beta_{0}}{\chi^{2}}, \quad g=\frac{\beta_{0}}{2 q \chi^{2}} .
$$

The functions $\gamma$ and $a$ can be expressed in terms of $f$ and its derivatives with respect to $\chi$ as

$$
\gamma=-q \chi f^{\prime}, \quad a=-q^{2}\left(f f^{\prime \prime}+\frac{3}{\chi} f f^{\prime}\right) .
$$

Then the last relation in (5) reduces to a third order linear ODE

$$
\chi\left(f f^{\prime}\right)^{\prime \prime}+9\left(f f^{\prime}\right)^{\prime}+\frac{15}{\chi} f f^{\prime}=\frac{6 p}{q^{3}},
$$

which is readily integrated to

$$
f= \pm \sqrt{c_{2}+f_{1} \chi^{2}+\frac{c_{1}}{\chi^{2}}+\frac{c_{0}}{\chi^{4}}}, \quad f_{1}=\frac{p}{4 q^{3}} .
$$

The remaining functions become

$$
\begin{aligned}
& a=\frac{q^{2}}{f^{2}}\left(\frac{c_{1}^{2}-4 c_{0} c_{2}}{\chi^{6}}-\frac{12 c_{0} f_{1}}{\chi^{4}}-\frac{6 c_{1} f_{1}}{\chi^{2}}-4 c_{2} f_{1}-3 f_{1}^{2} \chi^{2}\right), \\
& \gamma=\frac{q}{f}\left(-f_{1} \chi^{2}+\frac{c_{1}}{\chi^{2}}+\frac{2 c_{0}}{\chi^{4}}\right) .
\end{aligned}
$$

The observables can be written, up to a scaling of the parameters, in terms of $F$ and $G$ defined by

$$
\begin{aligned}
& F=4 q^{2} \chi^{4} f^{2}=c_{0}+c_{1} \zeta+c_{2} \zeta^{2}+g_{1} \zeta^{3}, \quad g_{1}=\frac{p}{q}, \quad \zeta=\chi^{2}, \\
& G=16 q^{2} \chi^{6} f^{2} a=c_{1}^{2}-4 c_{0} c_{2}-12 c_{0} g_{1} \zeta-6 c_{1} g_{1} \zeta^{2}-4 c_{2} g_{1} \zeta^{3}-3 g_{1}^{2} \zeta^{4} .
\end{aligned}
$$

To simplify matters the symplectic change of coordinates $\left(\theta, \phi, P_{\theta}, P_{\phi}\right) \rightarrow\left(\zeta, \phi, P_{\zeta}, P_{\phi}\right)$. gives the required result, up to scalings.

Alternatively (46) implies the relations

$$
G^{\prime}=-12 \frac{p}{q} F, \quad G=F^{2}-2 F F^{\prime \prime},
$$

which allow a direct check of $\{H, Q\}=0$. As proved in [8] this system does not exhibit any other conserved observable linear or quadratic in the momenta, and $(H, Q)$ are algebraically independent.

\section{Remarks:}

1. The limit $q=0$ is quite tricky: it is why we analyzed it separately in the previous section.

2. It is still possible to come back to the coordinate $\theta$ but the price to pay is the integration of the relation $\sqrt{\zeta / F} d \zeta=-d \theta$, which can be done using elementary functions for $c_{0}=0$. 


\section{The manifolds}

Let us now examine the global geometric aspects of the metric

$$
g=\frac{\zeta}{F} d \zeta^{2}+\frac{4 \zeta F}{G} d \phi^{2}, \quad \phi \in[0,2 \pi),
$$

taking into account the following observations:

1. The positivity constraints are $\zeta F(\zeta)>0$ and $G(\zeta)>0$. They define the endpoints of some interval $I$ for $\zeta$. In some cases, discussed in detail later on, one can obtain extensions beyond some of the end-points.

2. For the observables to be defined it is required that $F \geq 0 \quad \forall \zeta \in I$.

3. As already observed any point $\zeta_{0}$ with $F\left(\zeta_{0}\right) \neq 0$ and $G\left(\zeta_{0}\right)=0$ is a curvature singularity.

4. The point $\zeta=0$ is a curvature singularity for $F(0) \neq 0$ and $G(0) \neq 0$.

In order to have a complete description of all the possible integrable models, we will present them in three sets: the first one for $p=0$, the second one for $p>0$ and the third one for $p<0$.

\subsection{First set of integrable models}

For $p=0$ we have

$$
F=c_{0}+c_{1} \zeta+c_{2} \zeta^{2}=c_{2}\left(\zeta-\zeta_{1}\right)\left(\zeta-\zeta_{2}\right), \quad G=c_{1}^{2}-4 c_{0} c_{2}, \quad\left(c_{0}, c_{1}, c_{2}\right) \in \mathbb{R}^{3} .
$$

Theorem 6 In this set we have the following integrable models:

(i) Iff $c_{2}>0$ and $0<\zeta_{2}<\zeta$ the metric (48) is defined in $\mathbb{H}^{2}$ and

$$
\left\{\begin{array}{l}
H=\frac{1}{2} \frac{M_{1}^{2}+M_{2}^{2}-M_{3}^{2}}{\rho+\cosh u}+\frac{\alpha \sinh u \cos \phi+\beta}{\rho+\cosh u}, \quad u \in(0,+\infty), \\
Q=H M_{3}-\alpha M_{1}, \quad \rho=\frac{\zeta_{2}+\zeta_{1}}{\zeta_{2}-\zeta_{1}} \in(-1,+\infty)
\end{array}\right.
$$

(ii) Iff $c_{2}<0$ and $0<\zeta_{1}<\zeta<\zeta_{2}$ the metric (48) is defined in $\mathbb{S}^{2}$ and

$$
\left\{\begin{array}{l}
H=\frac{1}{2} \frac{L_{1}^{2}+L_{2}^{2}+L_{3}^{2}}{1+\rho \cos \theta}+\frac{\alpha \rho \sin \theta \cos \phi+\beta}{1+\rho \cos \theta}, \quad \theta \in(0, \pi), \\
Q=H L_{3}+\alpha L_{1}, \quad \rho=\frac{\zeta_{2}-\zeta_{1}}{\zeta_{2}+\zeta_{1}} \in(0,+1) .
\end{array}\right.
$$

(iii) Iff $c_{2}=0$ the metric (48) is defined in $\mathbb{R}^{2}$ and

$$
\left\{\begin{array}{l}
H=\frac{1}{2} \frac{P_{x}^{2}+P_{y}^{2}}{1+\rho^{2}\left(x^{2}+y^{2}\right)}+\frac{2 \alpha \rho^{2} x+\beta}{1+\rho^{2}\left(x^{2}+y^{2}\right)}, \quad(x, y) \in \mathbb{R}^{2}, \\
Q=H L_{z}-\alpha P_{y}, \quad \rho>0 .
\end{array}\right.
$$


In all three cases $\alpha$ and $\beta$ are free parameters.

(iv) All of these models are globally defined on their manifold.

Proof of (i): The positivity condition $G>0$ shows that $F$ has two real and distinct roots $\zeta_{1}<\zeta_{2}$, so we will write

$$
F=c_{2}\left(\zeta-\zeta_{1}\right)\left(\zeta-\zeta_{2}\right), \quad G=c_{2}^{2}\left(\zeta_{1}-\zeta_{2}\right)^{2} .
$$

Then imposing the positivity of $\zeta F$ one has to deal with the iff part of the proof by an enumeration of all possible cases for the triplet $\left(0, \zeta_{1}, \zeta_{2}\right)$, including the possibility of one $\zeta_{i}$ being zero. Taking into account the remarks given at the beginning of this Section, one concludes that for $c_{2}>0$, we must take $\zeta>\zeta_{2}>0$. The change of coordinates

$$
\zeta=\frac{\zeta_{2}-\zeta_{1}}{2}(\rho+\cosh u), \quad\left(\zeta_{2},+\infty\right) \rightarrow(0,+\infty), \quad \rho=\frac{\zeta_{2}+\zeta_{1}}{\zeta_{2}-\zeta_{1}} .
$$

brings the metric (48) to the form

$$
g=\frac{\zeta_{2}-\zeta_{1}}{2 c_{2}}(\rho+\cosh u)\left(d u^{2}+\sinh ^{2} u d \phi^{2}\right), \quad u \in(0,+\infty),
$$

which is conformal to the canonical metric on $\mathbb{H}^{2}$. Using the definitions (37) we obtain (50), up to scalings.

Proof of (ii): For $c_{2}<0$ positivity requires either $0<\zeta_{1}<\zeta<\zeta_{2}$ or $\zeta_{1}<\zeta<\zeta_{2}<0$. In both cases the change of coordinates

$$
\zeta=\frac{\zeta_{1}+\zeta_{2}}{2}(1+\rho \cos \theta), \quad\left(\zeta_{1}, \zeta_{2}\right) \rightarrow(\pi, 0), \quad \rho=\frac{\zeta_{2}-\zeta_{1}}{\zeta_{2}+\zeta_{1}}
$$

brings the metric (48) to one and the same form

$$
g=\frac{\zeta_{1}+\zeta_{2}}{2 c_{2}}(1+\rho \cos \theta)\left(d \theta^{2}+\sin ^{2} \theta d \phi^{2}\right), \quad \theta \in(0, \pi)
$$

which is conformal to the canonical metric on $\mathbb{S}^{2}$. Using the $s o(3)$ generators in $T^{*} \mathbb{S}^{2}$ one obtains (51), up to scalings.

Proof of (iii): For $c_{2}=0$ we have $G=c_{1}^{2}>0$.

If $c_{1}<0$ we can write $F=\left|c_{1}\right|\left(\zeta_{1}-\zeta\right)$ and positivity requires $\zeta \in\left(0, \zeta_{1}\right)$. If $\zeta_{1} \neq 0$ then $\zeta=0$ is a curvature singularity because $F(0)$ and $G(0)$ are not vanishing.

If $c_{1}>0$ we have $F=c_{1}\left(\zeta-\zeta_{1}\right)$. If $\zeta_{1}<0$ positivity requires either $\zeta>0$, but $\zeta=0$ is a curvature singularity, or $\zeta<\zeta_{1}$ and then $F$ is negative. If $\zeta_{1}=0$ the metric becomes

$$
g=\frac{1}{c_{1}}\left(d \zeta^{2}+4 \zeta^{2} d \phi^{2}\right)
$$

so to recover flat space we have to take $\widetilde{\phi}=2 \phi \in[0,2 \pi)$ and in $H$ appears a term of the form $\cos (\widetilde{\phi} / 2)$ which does not define a function in $\mathbb{R}^{2}$. Eventually, if $\zeta_{1}>0$ if we take $\zeta<0$ the point $\zeta=0$ is singular, so we are left with $\zeta>\zeta_{1}$. The change of coordinates

$$
\zeta=\zeta_{1}\left(1+\rho^{2} r^{2}\right), \quad \rho>0, \quad x=r \cos \phi, \quad y=r \sin \phi,
$$


brings the metric (48) to the form

$$
g=\frac{4 \zeta_{1}^{2} \rho^{2}}{c_{1}}\left(1+\rho^{2} r^{2}\right)\left(d x^{2}+d y^{2}\right), \quad(x, y) \in \mathbb{R}^{2} .
$$

Using the $e(3)$ Lie algebra generators $\left(P_{x}, P_{y}, L_{z}=x P_{y}-y P_{x}\right)$ we obtain (52), up to scalings.

Proof of (iv): In all of the three cases $H$ and $Q$ have been expressed in terms of globally defined quantities, up to conformal factors which are $C^{\infty}$ over their coordinates ranges.

The remaining cases are given by $p \neq 0$. It is convenient to rescale $F$ by $|p| / q$ and $G$ by $p^{2} / q^{2}$ in order to have

$$
F=\epsilon\left(\zeta^{3}+f_{0} \zeta^{2}+c_{1} \zeta+c_{0}\right), \quad \epsilon=\operatorname{sign}(p), \quad G=F^{2}-2 F F^{\prime \prime}, \quad G^{\prime}=-12 \epsilon F,
$$

and for the observables, up to scalings

$$
\left\{\begin{array}{l}
H=\frac{1}{2 \zeta}\left(F P_{\zeta}^{2}+\frac{G}{4 F} P_{\phi}^{2}\right)+\alpha \frac{\sqrt{F}}{\zeta} \cos \phi+\frac{\beta}{\zeta}, \\
Q=\epsilon P_{\phi}^{3}+2 H P_{\phi}-2 \alpha\left(\sqrt{F} \sin \phi P_{\zeta}+(\sqrt{F})^{\prime} \cos \phi P_{\phi}\right),
\end{array}\right.
$$

So the metric is still given by (48). We will denote by $\Delta_{\epsilon}$ the discriminant of $F$ according to the sign of $\epsilon$.

\subsection{Second set of integrable models}

For $p>0$ or $\epsilon=+1$ we have:

Theorem 7 The metric (48):

(i) Is defined, for $\Delta_{+}<0$, on $\mathbb{S}^{2}$ iff

$$
F=\left(\zeta-\zeta_{0}\right)\left(\zeta-\zeta_{1}\right)\left(\zeta-\zeta_{2}\right), \quad 0<\zeta_{0}<\zeta<\zeta_{1}<\zeta_{2}
$$

The integrable system, using the notations of Theorem 2 case (i), is

$$
\left\{\begin{array}{l}
H=\frac{1}{2 \zeta_{+}(u)}\left(P_{u}^{2}+\frac{D(u)}{s^{2} c^{2} d^{2}} P_{\phi}^{2}\right)+\alpha k^{2} \frac{s c d}{\zeta_{+}(u)} \cos \phi+\frac{\beta}{\zeta_{+}(u)} \\
Q=4 P_{\phi}^{3}+2 H P_{\phi}-\alpha\left(\sin \phi P_{u}+\frac{(s c d)^{\prime}}{s c d} \cos \phi P_{\phi}\right) \\
\zeta_{+}(u)=\rho+k^{2} \operatorname{sn}^{2} u, \quad u \in(0, K), \quad \rho=\frac{\zeta_{0}}{\zeta_{2}-\zeta_{0}}>0 .
\end{array}\right.
$$

(ii) Is defined, for $\Delta_{+}=0$, on $\mathbb{H}^{2}$ iff

$$
F=\left(\zeta-\zeta_{0}\right)\left(\zeta-\zeta_{1}\right)^{2}, \quad 0<\zeta_{0}<\zeta<\zeta_{1}
$$


The integrable system, using the notations of Theorem 2 case (ii), is

$$
\left\{\begin{array}{l}
H=\frac{1}{2 \zeta_{+}(u)}\left\{M_{1}^{2}+M_{2}^{2}-\left(1-\frac{3}{C^{2}}\right) M_{3}^{2}\right\}+\alpha \frac{T\left(1-T^{2}\right)}{\zeta_{+}(u)} \cos \phi+\frac{\beta}{\zeta_{+}(u)}, \\
Q=4 M_{3}^{3}+2 H M_{3}-\alpha\left(M_{1}-3 T \cos \phi M_{3}\right), \\
\zeta_{+}(u)=\rho+\tanh ^{2} u, \quad u \in(0,+\infty), \quad \rho=\frac{\zeta_{0}}{\zeta_{1}-\zeta_{0}}>0 .
\end{array}\right.
$$

(iii) Is not defined on any manifold for $\Delta_{+}>0$.

(iv) The systems (59) and (60) are globally defined on their manifold.

Proof of (i): The iff part results from a case by case examination of all possible orderings of the 4-plet $\left(0, \zeta_{0}, \zeta_{1}, \zeta_{2}\right)$, including the possibility of one of the $\zeta_{i}$ being zero. We will not give the full details which can be easily worked out, taking into account the remarks presented at the beginning of Section 5 . The reader can check that with $F=\left(\zeta-\zeta_{0}\right)(\zeta-$ $\left.\zeta_{1}\right)\left(\zeta-\zeta_{2}\right)$ and $0<\zeta_{0}<\zeta<\zeta_{1}<\zeta_{2}$, the polynomial $F$ is positive and vanishes at the end-points $\left(\zeta_{0}, \zeta_{1}\right)$ while $G$ is strictly positive. It follows that $\zeta=\zeta_{0}$ and $\zeta=\zeta_{1}$ are the poles of the manifold $\mathbb{S}^{2}$. Operating the same coordinates change as in Theorem 2 , case (i), one obtains (59).

Proof of (ii): The polynomial $G$ becomes $G=\left(\zeta_{1}-\zeta\right)^{3}\left(3 \zeta+\zeta_{1}-4 \zeta_{0}\right)$. The change of variable

$$
\zeta=\left(\zeta_{1}-\zeta_{0}\right)\left(\rho+\operatorname{th}^{2} u\right), \quad \zeta \in\left(\zeta_{0}, \zeta_{1}\right) \rightarrow u \in(0,+\infty), \quad \rho=\frac{\zeta_{0}}{\zeta_{1}-\zeta_{0}},
$$

transforms the observables, up to scalings, into

$$
\left\{\begin{array}{l}
H=\frac{1}{2 \zeta_{+}(u)}\left(P_{u}^{2}+\frac{1+3 T^{2}}{S^{2}} P_{\phi}^{2}\right)+\frac{\alpha}{\zeta_{+}(u)} T\left(1-T^{2}\right) \cos \phi+\frac{\beta}{\zeta_{+}(u)} \\
Q=4 P_{\phi}^{3}+2 H P_{\phi}-\alpha \sin \phi P_{u}-\alpha \frac{\left(1-3 T^{2}\right)}{T} \cos \phi P_{\phi}, \\
\zeta_{+}(u)=\rho+\tanh ^{2} u .
\end{array}\right.
$$

Using the relations (37) one gets (60).

Proof of (iii): Examining all the possible cases gives no manifold for the metric.

Proof of (iv): For the first model (resp. the second model) the result follows from Theorem 3 (resp. Theorem 4) and the observation that the conformal factor $\zeta_{+}(u)$ is $C^{\infty}$ on the interval $[0, K]$ (resp. $[0,+\infty))$ and never vanishes.

\subsection{Third set of integrable models}

It is given by $p<0$ or $\epsilon=-1$. It displays a richer structure and for clarity we will split up the description of the integrable systems into several theorems.

Theorem 8 The metric (48) for $\Delta_{-}<0$ is defined on $\mathbb{S}^{2}$ iff: 
(i) either $F=\left(\zeta-\zeta_{0}\right)\left(\zeta-\zeta_{1}\right)\left(\zeta_{2}-\zeta\right), \quad \zeta_{0}<\zeta_{1}<\zeta<\zeta_{2}\left(\zeta_{1}>0\right)$.

The change of coordinates

$$
\operatorname{sn}\left(u, k^{2}\right)=\sqrt{\frac{\zeta_{2}-\zeta}{\zeta_{2}-\zeta_{1}}}, \quad u \in(0, K), \quad k^{2}=\frac{\zeta_{2}-\zeta_{1}}{\zeta_{2}-\zeta_{0}} \in(0,1),
$$

gives for integrable system

$$
\left\{\begin{array}{l}
H=\frac{1}{2 \zeta_{-}(u)}\left(P_{u}^{2}+\frac{D(u)}{s^{2} c^{2} d^{2}} P_{\phi}^{2}\right)+\alpha \frac{k^{2} s c d}{\zeta_{-}(u)} \cos \phi+\frac{\beta}{\zeta_{-}(u)} \\
Q=-4 P_{\phi}^{3}+2 H P_{\phi}+\alpha\left(\sin \phi P_{u}+\frac{(s c d)^{\prime}}{s c d} \cos \phi P_{\phi}\right) \\
\zeta_{-}(u)=k^{2}\left(\rho-\operatorname{sn}^{2} u\right), \quad \rho=\frac{\zeta_{2}}{\zeta_{2}-\zeta_{1}}>1
\end{array}\right.
$$

This system is globally defined on $\mathbb{S}^{2}$.

(ii) or $F=\left(\zeta_{0}-\zeta\right)\left(\zeta-\zeta_{1}\right)\left(\zeta-\zeta_{2}\right)$ and $G(0)=0, \quad 0<\zeta<\zeta_{0}<\zeta_{1}<\zeta_{2}$.

The integrable system is

$$
\left\{\begin{aligned}
& H=\frac{1}{2} f\left(L_{1}^{2}+L_{2}^{2}\right)+\frac{1}{2}\left(\frac{h}{3 f}-\cos ^{2} \theta f\right) \frac{L_{3}^{2}}{\sin ^{2} \theta}+ \\
&+\alpha \frac{\sin \theta \sqrt{f}}{\left(\cos ^{2} \theta\right)^{1 / 3}} \cos \phi+\frac{\beta}{\left(\cos ^{2} \theta\right)^{1 / 3}} \\
& Q=-\frac{4}{9} L_{3}^{3}+2 H L_{3}+3 \alpha(\cos \theta)^{1 / 3}\left(\sqrt{f} L_{1}+(\sqrt{f})^{\prime} \cos \phi L_{3}\right)
\end{aligned}\right.
$$

where $f(\theta)=\hat{f}(\cos \theta)$ with

$$
\hat{f}(\mu)=\frac{\left(\frac{\zeta_{1}}{\zeta_{0}}-\mu^{2 / 3}\right)\left(\frac{\zeta_{2}}{\zeta_{0}}-\mu^{2 / 3}\right)}{\mu^{4 / 3}+\mu^{2 / 3}+1}, \quad \mu \in(-1,+1),
$$

and $h(\theta)=\hat{h}(\cos \theta)$ with

$$
\hat{h}(\mu)=-\mu^{2}+\frac{4}{3}\left(1+\frac{\zeta_{1}+\zeta_{2}}{\zeta_{0}}\right) \mu^{4 / 3}-2\left(\frac{\zeta_{1}+\zeta_{2}}{\zeta_{0}}+\frac{\zeta_{1} \zeta_{2}}{\zeta_{0}^{2}}\right) \mu^{2 / 3}+4 \frac{\zeta_{1} \zeta_{2}}{\zeta_{0}^{2}} .
$$

The parameter $\zeta_{0}$ is:

$$
\zeta_{0}=\frac{\zeta_{1} \zeta_{2}}{\left(\sqrt{\zeta_{1}}+\sqrt{\zeta_{2}}\right)^{2}}<\zeta_{1}
$$

This system exhibits a singular Hamiltonian flow.

Proof of (i): The change of variable (62) gives (63) by lengthy but straightforward computations. It is globally defined from Theorem 3 and because the conformal factor $\zeta_{-}(u)$ is $C^{\infty}$ and never vanishes on $[0, K]$. 
Proof of (ii): One has

$$
G(0)=\left(\zeta_{1}-\zeta_{2}\right)^{2} \zeta_{0}^{2}-2 \zeta_{1} \zeta_{2}\left(\zeta_{1}+\zeta_{2}\right) \zeta_{0}+\zeta_{1}^{2} \zeta_{2}^{2}
$$

Its vanishing determines uniquely $\zeta_{0}$ in terms of $\left(\zeta_{1}, \zeta_{2}\right)$ as given by (67). At this stage positivity requires $\zeta \in\left(0, \zeta_{0}\right)$. Let us make the change of variable $\zeta=\zeta_{0} \mu^{2 / 3}$. The metric becomes

$$
g=\frac{4}{9}\left\{\frac{d \mu^{2}}{\left(1-\mu^{2}\right) \hat{f}(\mu)}+3\left(1-\mu^{2}\right) \frac{\hat{f}(\mu)}{\hat{h}(\mu)} d \phi^{2}\right\}, \quad \mu \in(0,1) .
$$

All the functions in the metric are even functions of $\mu$ : we can therefore take $\mu \in(-1,+1)$ extending the metric beyond $\mu=0$. One can check that the points $\mu= \pm 1$ are "nuts" and therefore we get again for manifold $\mathbb{S}^{2}$. The change of variable $\mu=\cos \theta$ with $\theta \in(0, \pi)$ gives then for result (64).

As previously observed in the Remark of Subsection 3.3, for the Hamiltonian flow to be defined, the function $\widehat{f}$ must be differentiable for all $\mu \in[-1,+1]$ and this is not true for $\mu=0$ since $\widehat{f}$ is a function of $\mu^{2 / 3}$. Let us notice that no choice of the parameters $\zeta_{0}, \zeta_{1}$ and $\zeta_{2}$ allows to cure $\widehat{f}$ from this pathology.

Remark: The Theorem 8 case (i) does not describe appropriately the special case $\zeta_{0}=0$ for which elliptic functions are no longer required. Indeed the coordinates change

$$
\zeta=\frac{\zeta_{1}+\zeta_{2}}{2}-\frac{\zeta_{1}-\zeta_{2}}{2} \cos \theta, \quad\left(\zeta_{1}, \zeta_{2}\right) \rightarrow(\pi, 0)
$$

gives for the metric

$$
g=d \theta^{2}+\frac{\sin ^{2} \theta}{1+\sin ^{2} \theta G(\cos \theta)} d \phi^{2}
$$

with

$$
G(\mu)=\frac{3 \mu^{2}+4 \rho \mu+1}{4(\rho+\mu)^{2}}, \quad \rho=\frac{\zeta_{2}+\zeta_{1}}{\zeta_{2}-\zeta_{1}}>1 .
$$

The integrable system is

$$
\left\{\begin{array}{l}
H=\frac{1}{2}\left(L_{1}^{2}+L_{2}^{2}+(1+G(\cos \theta)) L_{3}^{2}\right)+\alpha \frac{\sin \theta}{\sqrt{U}} \cos \phi+\frac{\beta}{U}, \\
Q=-L_{3}^{3}+2 H L_{3}+2 \alpha \sqrt{U} L_{1}-\alpha \frac{\sin \theta}{\sqrt{U}} \cos \phi L_{3},
\end{array}=\rho+\cos \theta .\right.
$$

on which we recognize the Dullin-Matveev system [4].

Let us proceed to:

Theorem $9 \quad$ (a) The metric (48) for $\Delta_{-}=0$ is defined on $\mathbb{S}^{2}$ iff:

(i) either $F=\zeta^{2}\left(\zeta_{0}-\zeta\right), \quad 0<\zeta<\zeta_{0}$, and we have

$$
\left\{\begin{array}{l}
H=\frac{1}{2}\left(L_{1}^{2}+L_{2}^{2}+4 L_{3}^{2}\right)+\alpha \sin \theta \cos \phi+\frac{\beta}{\cos ^{2} \theta}, \quad \theta \in(0, \pi), \\
Q=-4 L_{3}^{3}+2 H L_{3}+\alpha\left(\cos \theta L_{1}-2 \sin \theta \cos \phi L_{3}\right)
\end{array}\right.
$$

which is the Goryachev-Chaplygin top, globally defined for $\beta=0$. 
(ii) or $F=\left(\zeta-\zeta_{1}\right)^{2}\left(\zeta_{0}-\zeta\right)$ and $G(0)=0, \quad 0<\zeta<\zeta_{0}$.

The integrable system (with $\zeta_{0}=\zeta_{1} / 4$ ) has the form (64) with the functions

$$
\hat{f}(\mu)=\frac{\left(4-\mu^{2 / 3}\right)^{2}}{\mu^{4 / 3}+\mu^{2 / 3}+1}, \quad \hat{h}(\mu)=\left(4-\mu^{2 / 3}\right)^{3}, \quad \mu \in(-1,+1) .
$$

This system exhibits a singular Hamiltonian flow.

(b) The metric (48) for $\Delta_{-}=0$ is defined on $\mathbb{H}^{2}$ iff:

$$
F=\left(\zeta-\zeta_{1}\right)^{2}\left(\zeta_{0}-\zeta\right), \quad 0<\zeta_{1}<\zeta<\zeta_{0}
$$

The integrable system, in the notations of Theorem 2, case (ii), is

$$
\left\{\begin{array}{l}
H=\frac{1}{2 \zeta_{-}(u)}\left\{M_{1}^{2}+M_{2}^{2}-\left(1-\frac{3}{C^{2}}\right) M_{3}^{2}\right\}+\alpha \frac{T\left(1-T^{2}\right)}{\zeta_{-}(u)} \cos \phi+\frac{\beta}{\zeta_{-}(u)} \\
Q=-4 M_{3}^{3}+2 H M_{3}+\alpha\left(M_{1}-3 T \cos \phi M_{3}\right) \\
\zeta_{-}(u)=\rho-\tanh ^{2} u, \quad u \in(0,+\infty), \quad \rho=\frac{\zeta_{0}}{\zeta_{0}-\zeta_{1}}>1
\end{array}\right.
$$

It is globally defined on $\mathbb{H}^{2}$.

Proof of (a)(i): We have $F=\zeta^{2}\left(\zeta_{0}-\zeta\right)$ and $G=\zeta^{3}\left(4 \zeta_{0}-3 \zeta\right)$ and $\zeta \in\left(0, \zeta_{0}\right)$ from positivity. Taking for new variable $\theta$ such that $\zeta=\zeta_{0} \cos ^{2} \theta$ we get for the metric

$$
g=4\left(d \theta^{2}+\frac{\sin ^{2} \theta}{1+3 \sin ^{2} \theta} d \phi^{2}\right), \quad \theta \in(0, \pi / 2) .
$$

As it stands the manifold is $P^{2}(\mathbb{R})$ (see [1][p. 268]), but we can take $\theta \in(0, \pi)$ extending the manifold to $\mathbb{S}^{2}$ with poles for $\theta=0$ and $\theta=\pi$. The observables can be transformed into (71) and we recover the Goryachev-Chaplygin top.

Proof of (a)(ii): In this case we have $G(0)=\zeta_{1}^{3}\left(\zeta_{1}-4 \zeta_{0}\right)$ which fixes $\zeta_{0}=\zeta_{1} / 4$. The argument then proceeds as in the proof of Theorem 8 , case (ii).

Proof of (b): The proof is identical to the one for Theorem 7, case (ii), except for the change of coordinates, which is now

$$
\zeta=\zeta_{0}-\left(\zeta_{0}-\zeta_{1}\right) \tanh ^{2} u \quad\left(\zeta_{0}, \zeta_{1}\right) \quad \rightarrow \quad(0,+\infty)
$$

and leads to $(73)$.

Theorem 10 The metric (48) for $\Delta_{-}>0$ is defined on $\mathbb{S}^{2}$ iff:

$$
F=\left(\zeta_{0}-\zeta\right)\left(\zeta-\zeta_{1}\right)\left(\zeta-\overline{\zeta_{1}}\right) \text { and } G(0)=0, \quad 0<\zeta<\zeta_{0}
$$

The integrable system is of the form (64) with the functions

$$
\hat{f}(\mu)=\frac{\left(\mu^{2 / 3}-\frac{\zeta_{1}}{\zeta_{0}}\right)\left(\mu^{2 / 3}-\frac{\bar{\zeta}_{1}}{\zeta_{0}}\right)}{\mu^{4 / 3}+\mu^{2 / 3}+1}, \quad \mu \in(-1,+1),
$$


and

$$
\hat{h}(\mu)=-\mu^{2}+\frac{4}{3}\left(1+\frac{\zeta_{1}+\bar{\zeta}_{1}}{\zeta_{0}}\right) \mu^{4 / 3}-2\left(\frac{\zeta_{1}+\bar{\zeta}_{1}}{\zeta_{0}}+\frac{\left|\zeta_{1}\right|^{2}}{\zeta_{0}^{2}}\right) \mu^{2 / 3}+4 \frac{\left|\zeta_{1}\right|^{2}}{\zeta_{0}^{2}} .
$$

The value of $\zeta_{0}$ is

$$
\zeta_{0}=\frac{\left|\zeta_{1}\right|^{2}}{\zeta_{1}+\bar{\zeta}_{1}+2\left|\zeta_{1}\right|}>0
$$

This system, for generic parameters, exhibits a singular Hamiltonian flow.

Proof: We have

$$
G(0)=\left(\zeta_{1}-\bar{\zeta}_{1}\right)^{2} \zeta_{0}^{2}-2\left(\zeta_{1}+\bar{\zeta}_{1}\right)\left|\zeta_{1}\right|^{2} \zeta_{0}+\left|\zeta_{1}\right|^{4}
$$

Its vanishing gives two roots for $\zeta_{0}$, but only (77) is positive. The subsequent analysis is identical to that already given in the proof of Theorem 8, case (ii). For some special values of the parameters $\widehat{f}$ may reduce to a constant, as for the Goryachev top examined just below.

To conclude, let us examine the explicitly known integrable systems, with a metric defined in $\mathbb{S}^{2}$ and with a cubic observable already given in the literature:

1. The Goryachev-Chaplygin top given by Theorem 9, case (a)(i).

2. The Dullin-Matveev top [4] given in the remark after the proof of Theorem 8 .

3. If we restrict, in Theorem 10, the parameters according to

$$
\zeta_{0}=-\left(\zeta_{1}+\bar{\zeta}_{1}\right)>0 \quad \text { and } \quad \zeta_{0}=\left|\zeta_{1}\right|, \quad \Longrightarrow \quad \widehat{f}=1, \quad \widehat{h}=4-\mu^{2}
$$

we recover the Goryachev top

$$
\left\{\begin{array}{l}
H=\frac{1}{2}\left(L_{1}^{2}+L_{2}^{2}+\frac{4}{3} L_{3}^{2}\right)+\alpha \frac{\sin \theta}{\left(\cos ^{2} \theta\right)^{1 / 3}} \cos \phi+\frac{\beta}{\left(\cos ^{2} \theta\right)^{1 / 3}}, \\
Q=-\frac{4}{9} L_{3}^{3}+2 H L_{3}+3 \alpha(\cos \theta)^{1 / 3} L_{1} .
\end{array}\right.
$$

However, due to the "equatorial" singularities $(\theta=\pi / 2)$ of the potential, this system is globally defined only in the trivial case $\alpha=\beta=0$.

4. The two new examples given by Tsiganov in [10] are not defined on a manifold. All of the previously known examples belong to the third set with $p<0$.

\section{Conclusion}

We have exhaustively and explicitly constructed all of the integrable models, on two dimensional manifolds, for Selivanova's models characterized by the following form of the observables

$$
\left\{\begin{array}{l}
H=\frac{1}{2}\left(P_{\theta}^{2}+a(\theta) P_{\phi}^{2}\right)+f(\theta) \cos \phi+g(\theta) \\
Q=p P_{\phi}^{3}+q\left(P_{\theta}^{2}+a(\theta) P_{\phi}^{2}\right) P_{\phi}+\chi(\theta) \sin \phi P_{\theta}+(\beta(\theta)+\gamma(\theta) \cos \phi) P_{\phi}
\end{array}\right.
$$


The main point which stems from our work is that the coordinates choice is of the utmost delicacy since it determines the structure of the differential equations to be solved eventually. The same difficulty must be overcome when looking for Einstein metrics with symmetry: the Einstein equations reduce to coupled ODE and finding exact solutions relies on an adapted choice of coordinates which may simplify or even linearize the differential system to be integrated.

Acknowledgments: We are greatly indebted to K. P. Tod for his kind and efficient help in the analysis of the metric singularities of Section 5, and to the Referee for several improvements and corrections induced by his observations.

\section{References}

[1] A. L. Besse, Einstein manifolds, Classics in Mathematics, Springer-Verlag, Berlin Heidelberg New-York (2002).

[2] A. V. Bolsinov, V. V. Kozlov and A. T. Fomenko, Russ. Math. Surv., 50 (1995) 473-501.

[3] G. Darboux, Leçons sur la théorie générale des surfaces, 3e partie, Chelsea Publishing Company (1972).

[4] H. R. Dullin and V. S. Matveev, Math. Research Lett., 11 (2004) 715-722.

[5] G. Gibbons and S. Hawking, Commun. Math. Phys., 66 (1979) 291-310.

[6] K. Kiyohara, Math. Ann., 320 (2001) 487-505.

[7] S. V. Kovalevskaya, Acta Math., 12 (1889) 177-232.

[8] E. N. Selivanova, Commun. Math. Phys., 207 (1999) 641-663.

[9] E. N. Selivanova, Ann. Global Anal. Geom., 17 (1999) 201-219.

[10] A. V. Tsiganov, J. Phys. A: Math. Gen., 38 (2005) 921-927. 